\title{
Automatisierte Modellierung von akademischen Wissensdomänen als Methode zum innovativen Wissensmanagement
}

https://doi.org/10.1515/iwp-2019-2071

Zusammenfassung: Die Modellierung von akademischen Wissensdomänen, deren Wissensinhalte sich schnell verändern, ist wegen des großen Aufwandes, ständiger Anpassungen und großer Datenmengen eine Herausforderung. Dieser Artikel stellt eine Lösung für eine neue, epistemologische Domänenmodellierung vor, die auf der Theorie menschlicher mentaler Modellbildung basiert und es erlaubt, akademische Wissensdomänen abzubilden, die ständigen Veränderungen unterworfen sind. Diese neue Art der Domänenmodellierung erweitert das vorhandene technologische Spektrum der Wissensmodellierung (knowledge engineering) um eine neue Methodenklasse und schließt die bisher bestehende konzeptuelle Lücke in der Vermittlung zwischen maschineller Wissensaufbereitung und der menschlichen Nutzung. Menschliches Wissen zeichnet sich durch eine kontextabhängige Vernetzung von Informationen aus. Diese Vernetzungen sind assoziativer Natur. Entsprechend dieser grundlegenden Funktionsweise menschlichen Wissens analysiert das validierte Textanalyseprogramm T-MITOCAR (Text Model Inspection Trace of Concepts and Relations) die in Text enthaltenen semantischen Wissensstrukturen computerlinguistisch und bildet sie als Assoziationsnetze (Landkarten des Wissens) ab. Die resultierenden Wissenslandkarten ähneln in ihrem Erscheinungsbild einer Mindmap. Ziel dabei ist es, eine adäquate Re-Repräsentation menschlichen Wissens auf der Grundlage von Text bereitzustel-

\footnotetext{
*Kontaktperson: Inga Kampmann, M.Sc., Pädagogische Psychologie, Martin-Luther-Universität Halle-Wittenberg, Franckeplatz 1, Haus 5, 06110 Halle (Saale),

E-Mail: inga.kampmann@paedagogik.uni-halle.de

Dr. phil. Inka Hähnlein, Pädagogische Psychologie, Martin-LutherUniversität Halle-Wittenberg, Dachritzstraße 12, 06108 Halle (Saale), E-Mail: inka.haehnlein@paedagogik.uni-halle.de

Prof. Dr. phil. habil. Pablo Pirnay-Dummer, Pädagogische

Psychologie, Martin-Luther-Universität Halle-Wittenberg,

Franckeplatz 1, Haus 5, 06099 Halle (Saale),

E-Mail: pablo.pirnay-dummer@paedagogik.uni-halle.de
}

len. Die Methoden können ebenfalls ganze Textkorpora in einem zweiten Schritt zu einer Wissensdomänenlandkarte aggregieren. Mittels dieser Technologien kann eine vollständige akademische Wissensdomäne automatisch als Wissenslandkarte abgebildet werden. Solche Wissensdomänenmodelle können von Institutionen mit schnelllebigen Wissensdomänen, wie z.B. Universitäten und Fachinformationsdiensten (FDI), als Instrumente des innovativen Wissens- und Informationsmanagements eingesetzt werden. Die Methoden ermöglichen Bibliotheken beispielsweise, aus verschiedenen Textkorpora automatisch je eine Wissenslandkarte zu generieren, diese $\mathrm{zu}$ vergleichen und inhaltliche Überschneidungen der zugrunde liegenden Wissensdomänen zu identifizieren. Veränderungen an der Wissensdomäne werden überblicksartig analysierbar und Literaturempfehlungen lassen sich auf der Grundlage von bereits Geschriebenen ausgeben.

Deskriptoren: Automatische Sprachverarbeitung, Verarbeitung natürlicher Sprache, Software, Wissensnetz, Wissensmanagement

\section{Automated modelling of academic knowledge domains as a method for innovative knowledge management}

Abstract: The knowledge content of academic knowledge domains is subject to constant change. Modelling these rapidly evolving domains of knowledge is a challenge due to the complexity, constant adaptations, and large amounts of data. This article presents a new epistemological approach to domain modelling based on the theory of human mental modelling. It allows to map these dynamic domains of knowledge. This approach extends the existing technological spectrum of knowledge engineering by a new class of methods. It closes the conceptual gap in the transfer between machine knowledge processing and human use. Human knowledge is characterized by a contextdependent networking of information. These networks are associative in nature. According to this basic functionality of human knowledge, the validated text analysis program T-MITOCAR (Text Model Inspection Trace of Concepts and 
Relations) analyses the semantic knowledge structures contained in text in a computer-linguistic way and maps them as association networks (maps of knowledge). The resulting knowledge maps resemble a mind map in their appearance. The aim is to provide an adequate re-representation of human knowledge on the basis of text. In a second step, these methods can also aggregate entire text corpora into a map of the knowledge domain. Using these technologies, a complete academic knowledge domain can be modeled automatically as a knowledge map. Such knowledge domain models can be used as instruments of innovative knowledge and information management by institutions with fast-moving knowledge domains, such as universities and specialized information services. The methods enable libraries, for example, to automatically generate a knowledge map from different text corpora, to compare them and to identify overlapping contents of the underlying knowledge domains. Changes in the knowledge domain can be analyzed in an overview-like manner and literature recommendations can be issued based on what has previously been written.

Descriptors: Automatic language processing, Natural language processing, Software, Knowledge network, Knowledge management

\section{Modélisation automatisée des domaines de connaissan- ces académiques comme méthode de gestion innovatrice des connaissances}

Résumé: Modéliser des domaines de connaissances académiques dont le contenu des connaissances change rapidement est un défi en raison de la complexité, de l'adaptation constante et des grandes quantités de données. Cet article présente une solution pour une nouvelle modélisation du domaine épistémologique basée sur la théorie de la modélisation mentale humaine qui permet de cartographier les domaines de connaissances académiques soumis à des changements constants. Ce nouveau type de modélisation de domaine élargit le spectre technologique existant de l'ingénierie du savoir par une nouvelle classe de méthodes et comble le fossé conceptuel dans la médiation entre le traitement du savoir machine et l'utilisation humaine. Le savoir humain se caractérise par une mise en réseau de l'information en fonction du contexte. Ces réseaux sont de nature associative. En fonction de cette fonctionnalité de base du savoir humain, le programme d'analyse de texte validé T-MITOCAR (Text Model Inspection Trace of Concepts and Relations) analyse les structures de connaissances sémantiques contenues dans le texte de façon informatique-linguistique et les cartographie sous forme de réseaux d'associations (cartes de connais- sances). Les cartes des connaissances qui en résultent ressemblent à une carte mentale dans leur apparence. L'objectif est d'assurer une représentation adéquate de la connaissance humaine sur la base du texte. Dans un deuxième temps, les méthodes peuvent également agréger des corpus de textes entiers dans une carte du domaine de connaissances. Grâce à ces technologies, un domaine de connaissances académiques complet peut être automatiquement cartographié comme une carte des connaissances. Ces modèles de domaines de connaissances peuvent être utilisés comme instruments de gestion novatrice des connaissances et de l'information par des institutions dont les domaines de connaissances évoluent rapidement, comme les universités et les services d'information spécialisés (IED). Ces méthodes permettent par exemple aux bibliothèques de générer automatiquement une carte des connaissances à partir de différents corpus de textes, de les comparer et d'identifier les chevauchements de contenus des domaines de connaissances sous-jacents. Les changements dans le domaine des connaissances peuvent être analysés de manière synthétique et des recommandations documentaires peuvent être formulées sur la base d'informations écrites préalables.

Descripteurs: Traitement automatique des langues, Traitement du langage naturel, Logiciels, Réseau de connaissances, Gestion des connaissances

\section{Wissen und die Wissensdomäne}

\begin{abstract}
„Das Wissen der Menschheit verdoppelt sich alle fünf Jahre, während die Hälfte davon in ca. drei Jahren wieder veraltet, so schätzen Experten. Jede Minute entsteht eine neue chemische Formel, alle drei Minuten ein neuer physikalischer Zusammenhang, alle fünf Minuten eine neue medizinische Erkenntnis“ (Glaser, 2002, S. 123).
\end{abstract}

Wissen ist nicht nur dynamisch, es ist auch immer das Wissen über etwas und steht grundsätzlich in Bezug zueinander (Mertins, 2009). Wissen bleibt ohne eine sinnvolle Einordnung in seinen Kontext abstrakt und unkonkret. Es ist erforderlich, Wege zu finden, mittels derer sich das Wissen in einen greifbaren und konkreten Zusammenhang bringen lässt, um es zu beschreiben. Ein Weg, um Wissen zu systematisieren, ist die Erstellung eines Wissensdomänenmodells. Ein Wissensdomänenmodell ist ein modellhaftes und daher übersichtliches Abbild eines Wissensgebiets. Die Beschreibung einer Wissensdomäne, also eines konkreten Wissensgebietes, ermöglicht es, in der Praxis - genauer: der Wissensverwendung - eine Beschreibung des Wissens zu erarbeiten und festzulegen 
(Mertins, 2009). Ist ein solches Modell erst einmal erstellt, eröffnen sich damit einige weitere Anwendungsmöglichkeiten des Wissensmanagements. Ein Wissensdomänenmodell ermöglicht die Fokussierung von Wissensmanagementaktivitäten auf die wesentlichen Inhalte und damit auf das Know-how in Institutionen (Orth, Finke, Voigt, 2008).

„Wissensmanagement setzt Instrumente auf vielen verschiedenen Interventionsebenen ein, um auf individueller und kollektiver Ebene den Umgang mit Wissen zu verbessern, mit dem Ziel, schließlich die durch die Wissensmanagementstrategie festgelegten Ziele zu erreichen“ (Maier, 2002, S. 47).

Die Ziele, die mittels des Wissensdomänenmodells im Rahmen des Wissensmanagements erreicht werden können, hängen von der Wahl der Methode ab, mit der die Domäne modelliert wird. Laut Bimba et al. (2016) existieren vier verschiedene, gängige Arten Wissen zu modellieren. Es gibt die linguistische Wissensmodellierung, Wissensmodellierung durch Experten, ontologische Ansätze und die kognitive Wissensmodellierung. In diesem Artikel wird zunächst kurz auf den ontologischen Ansatz und anschließend auf die computerlinguistische Modellierungsmethode T-MITOCAR Artemis eingegangen, die am ehesten dem kognitiven Ansatz der Wissensmodellierung zuzuordnen ist, mit starken Brücken in die linguistische Wissensmodellierung.

Die verschiedenen Ansätze haben das Ziel gemeinsam, die Inhalte der Domäne bei der Modellierung in einer spezifischen Ordnung zu strukturieren.

Die Schwierigkeit besteht demnach nicht in der Bereitstellung der Information, sondern ganz im Gegenteil, in der Identifikation und Zusammenfassung der relevanten Informationen (Pawlowsky, 2019).

Eine maschinelle, automatisierte Domänenmodellierung, z. B. eine ontologische- oder computerlinguistische Modellierung, ist ein Weg, um große Mengen an Informationen, die in Wissensdomänen enthalten sind und die in Form von Text vorliegen, verarbeiten und abbilden zu können.

\section{Ontologische Ansätze der Domänenmodellierung}

In den vergangenen Jahren haben sich verschiedene Methoden und Ansätze entwickelt, um Wissen automatisiert abzubilden und zu systematisieren (Bimba et. al., 2016).

Zuvorderst ist hier das Semantic Web zu nennen, das auch als Web 3.0 bezeichnet wird (Hitzler, Krötzsch, Ru- dolph, \& Sure, 2007). Bei dieser Form der Webontologie werden Begriffen spezifische Informationen zugeordnet, um sie somit eindeutig und für Maschinen lesbar zu machen (Villa, Athanasiadis, \& Rizzoli, 2009). Im Rahmen dieser Ansätze werden Begriffe manuell von Experten mit Kontextinformationen angereichert. Denn während Menschen für ihr Schlussfolgern automatisch Kontext als Informationsquellenheranziehen, brauchen Maschinen zusätzliche Informationen, die einer Informationseinheit, z. B. einem Wort, eindeutig zugeordnet sind. Als Produkt entsteht ein Konsensmodell eines Feldes, welches modellimmanente Widersprüche bereinigt und daher den Anspruch einer Ontologie in sich trägt: Wissen, das gültig ist, wird in einem formalen Repräsentationssystem so abgebildet, dass vor allem maschinelle Schlussfolgerungsprozesse damit möglich werden (Pirnay-Dummer, 2012).

Der Einsatz von Webontologien ist dann sinnvoll, wenn objektive Wissensverzeichnisse angelegt werden sollen. Also dann, wenn ein Begriff einer spezifischen, definierten Bedeutung zugeordnet wird, die nicht vom Dafürhalten einzelner abhängt und die weitgehend widerspruchsfrei konkrete oder abstrakte Objekte als Sammlung relevant erachteter Eigenschaften mit relationalen Strukturen verbindet. Wenngleich fortgeschrittene Ontologien unter Umständen mit Verfahren des Machine-Learnings irgendwann teilautomatisiert erweitert werden können (Gangemi, 2003; Maedche, Pekar \& Staab, 2002), so bedarf es immer noch eines eher in Arbeitsjahren $\mathrm{zu} \mathrm{Bu}$ che schlagenden Aufwands, die erforderliche Struktur zunächst manuell aufzubauen.

Akademische Wissensdomänen unterscheiden sich allerdings je nach Standort, Modellierungsanlass und Bildungskontext, was standort- und sogar personenspezifische Ontologien erforderte, um das zum Beispiel in der Lehre tatsächlich eingesetzte Wissen abzubilden.

\section{Expertisespezifität und epistemischer Zugang}

In der akademischen Landschaft existieren de facto viele verschiedene Wissensmodelle parallel und sind lokal an ihren Universitäten oder Bibliotheken angesiedelt und zusätzlich ständigen Veränderungen unterworfen. Angesichts dieser spezifischen Eigenschaften von akademischen Wissensdomänen sind ontologische Ansätze für deren Modellierung nur dann geeignet, wenn die Ressourcen für den individuellen, manuellen Aufbau zur Verfügung stehen. Der in diesem Artikel vorgestellte Ansatz zur Modellierung basiert auf der Theorie mentaler Modelle (John- 
son-Laird, 1983; Seel, 1991; Johnson et. al. 2002; Schnotz \& Preuss, 1997; Strasser, 2010) und bildet Wissen mittels eines epistemologischen und heuristischen Vorgehens ab.

Um die Möglichkeit zu schaffen, im Rahmen eines effizienten Informationsmanagements, Wissensdomänen flächendeckend zu modellieren und einzusetzen, begegnet die Technologie um T-MITOCAR dieser Herausforderung mittels eines automatisierten, heuristischen Vorgehens, welches zugleich in seiner Struktur und Logik näher an menschlicher Wissensrepräsentation orientiert ist und weniger an maschineller Verarbeitung. Die Verwendung einer kognitiven, psycholinguistischen Semantik zur Extraktion von Wissensstrukturen aus Text schafft Abbilder von Wissen, die zwar noch maschinenverarbeitbar sind, deren Aufrechterhaltung von Ambiguität und Kontext jedoch der menschlichen Informationsverarbeitung deutlich nähersteht (Pirnay-Dummer, 2015a, 2015b). Die Nähe dieses Abbilds soll Menschen den Zugang erleichtern, gerade dann, wenn die Strukturen im technischen System zur Mensch-Maschine-Interaktion verwendet werden sollen. Die Heuristik selbst kommt ganz ohne manuellen Analyseaufwand aus und kann daher schneller als ontologische Ansätze der Dynamik von sich veränderndem Wissen gerecht werden. Die Technologie erlaubt es, in kurzer Zeit große Domänenmodelle mit vergleichsweise minimalem zeitlichem Aufwand zu erstellen, wobei eine aufgeräumte Digitalisierung von Text eine notwendige Voraussetzung dafür ist. Das Vorgehen ermöglicht unter anderem universitäts- bzw. bibliothekseigene Domänen zu modellieren.

Dabei werden die sich hinter den Texten der Literatur befindlichen Wissensmodelle einer Domäne mittels T-MITOCAR (Akronym für (Text Model Inspection Trace of Concepts and Relations) analysiert und mit der Erweiterung Artemis - zu übergeordneten Landkarten des Wissens zusammengefasst und dargestellt.

Kommt neue Literatur dazu, oder entfällt Literatur, können die entsprechenden Texte umgehend gelöscht oder aber neue Texte zum Modell hinzugefügt werden. Auf diesem Weg ist es jederzeit möglich, Anpassungen am Modell vorzunehmen und somit der Dynamik von sich veränderndem Wissen gerecht zu werden. Effizienz und Flexibilität dieses Vorgehens eröffnen Spielräume zur Modellierung, die vorher nicht in dieser Art zugänglich waren. Auf diese Weise entsteht ein Wissensnetzwerk. Was sich inhaltlich und strukturell ähnelt, wird auch eng beisammen verortet. Hierfür werden semantische Clusteranalysen auf den zuvor identifizierten Einzelmodellen durch die Software durchgeführt. Es können Ähnlichkeiten, Unterschiede und Veränderungen in Wissensdomänen identifiziert und quantifizierend abgebildet werden.
Für große Institutionen wie Universitäten, Bibliotheken und FDIs, deren Wissensinhalte sich, von dynamischen Faktoren bedingt, verändern und die dieses Wissen gleichzeitig auch für die Nutzung durch Menschen aufbereiten möchten und nicht nur die maschinelle Verarbeitung bereithalten müssen, bietet eine derartige epistemologische Domänenmodellierung neue, innovative Möglichkeiten zur Vernetzung von menschlicher und maschineller Wissensrepräsentation. Denn Menschen interpretieren Wissen stets epistemologisch auf der Basis der eigenen Wissensbestandteile (Seel, 1991).

\section{Computer-linguistische Heuristik zur Modellierung einzelner Wissensquellen hinter Texten}

Ein genauerer Blick auf die T-MITOCAR-Technologie soll den Prozess der Domänenmodellierung deutlicher machen:

T-MITOCAR basiert auf der Theorie mentaler Modelle (Johnson-Laird, 1983; Seel, 1991; Schnotz, 1994; PirnayDummer, Ifenthaler \& Seel, 2012; Pirnay-Dummer \& Seel, 2018), der Sprach- und Assoziationspsychologie (Lewin, 1922; Strasser, 2010; Jonassen \& Cho, 2008) und der formalen Semantik und Syntax als Teilbereiche der Linguistik und allgemeinen Sprachwissenschaft (Bach, 1968; Montague, 1974; Helbig, 2006).

Die Technologie fußt auf einem alten Theorem der Assoziations- und Sequenzforschung, welches besagt, dass Syntax und Semantik abhängig voneinander sind. Was im Individuum eng assoziiert ist, wird in Text von Strukturen der Syntax auch nahgebunden externalisiert (z.B. Smith, 1894; Wells, 1911). T-MITOCAR erlaubt es, Fließtext sehr schnell computerlinguistisch zu analysieren und daraus ein Modell zu konstruieren, das nicht nur Informationen darüber enthält, welche Begriffe in dem Text enthalten sind, sondern auch, wie stark diese Begriffe miteinander assoziiert sind (siehe Abb. 2). Bedeutung erhält dabei jedoch nicht die individuelle Assoziation und schon gar nicht die einzelne Begriffshäufigkeit, sondern das Netz, welches durch sie konstituiert wird. Eine einzelne Assoziation und auch einzelne Konzepte werden als zusammenhängende Einheiten also erst durch den Netzkontext mit Bedeutung versehen. Für die Analyse soll der einzugebende Text mindestens 350 Wörter enthalten.

Die Modellbestimmung verläuft immer nach dem gleichen Schema: Zuerst erfolgt die Texteingabe und danach die computerlinguistische Modellierung (Pirnay-Dummer, 


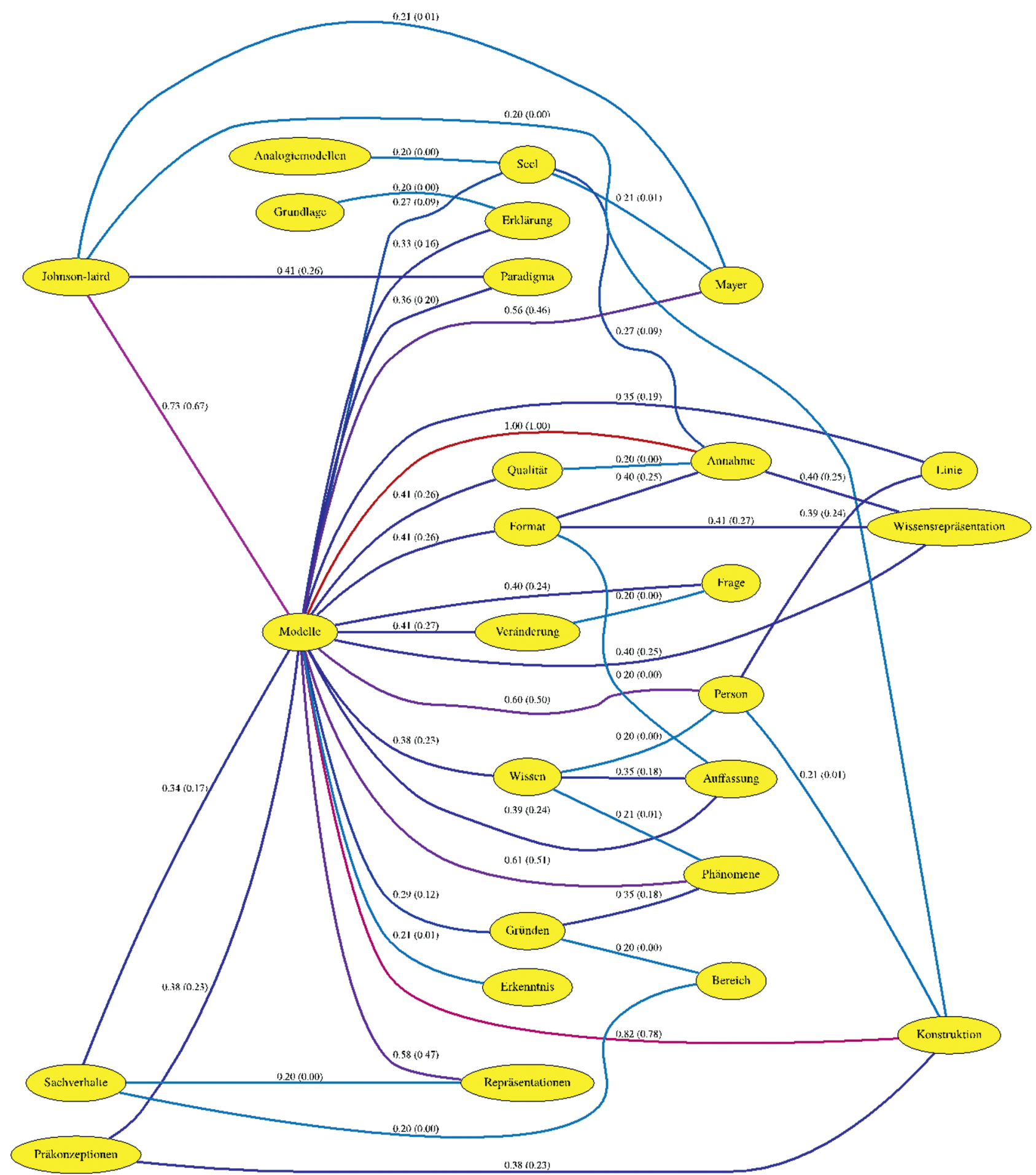

Abbildung 1: T-MITOCAR Assoziationsnetz über Einzeltext.

2006, 2010a, 2010 b, 2014, 2015a, 2015b). Das Produkt dieser Analyse, das Assoziationsnetz, kann nach der Modellierung als Graph gezeichnet und ausgegeben werden. Solch ein Graph ist in Abbildung 1 dargestellt. Das Beispiel zeigt ein Assoziationsnetz, über einen Text zur mentalen Modellbildung, von Seel (2003).
Das aus der T-MITOCAR Analyse resultierende semantische Wissensnetz besteht aus Knoten (Konzepten/Begriffen) und Kanten (Verbindungen) und ist damit formal ein Graph. Schnittstellen $\mathrm{zu}$ anderen semantischen Repräsentationsweisen sind somit vergleichsweise einfach zu realisieren. Konzepte werden miteinander auf proposi- 
tional-assoziative Weise verbunden und sind im Text unterschiedlich stark miteinander assoziiert. Der Grad der Assoziation wird relativ zu den Assoziationen des einzelnen Textes ermittelt ist einerseits über die Farbe der Kanten kodiert, wobei die stärksten Assoziationen rot und schwache Assoziationen hellblau eingezeichnet sind. Zum anderen gibt auch die Zahl zwischen 0 und 1 die Assoziationsstärke der beiden angrenzenden Konzepte in diesem Text an (1 ist der stärkste Assoziationsgrad zwischen zwei Konzepten in diesem Text). Es werden dabei keine textexternen Informationen herangezogen. Verarbeitet wird nur, was der angelegte Text auch wirklich beinhaltet. (Pirnay-Dummer \& Kampmann, 2018). Die Assoziationsstärken sind spezifisch für den analysierten Text. Wurden mehrere Texte analysiert, ist es möglich die verschiedenen Assoziationsnetze mittels T-MITOCAR automatisch im Hinblick auf ihre strukturelle und semantische Ähnlichkeit miteinander zu vergleichen. Bei diesem Vergleich kommen sieben verschiedene graphentheoretische Vergleichsmaße zum Einsatz (Tittmann, 2010), die über die Jahre als besonders wesentlich für menschliches Lernen identifiziert wurden. Es handelt sich um vier strukturelle Ähnlichkeitsmaße (surface, graphical, gamma und structural matching) und drei semantische Ähnlichkeitsmaße (concept, propositional und balanced semantic matching), deren Werte zwischen 0 und 1 liegen (Pirnay-Dummer, 2006, 2010, 2015a, Pirnay-Dummer \& Ifenthaler, 2010; Pirnay-Dummer, Ifenthaler \& Spector, 2010). Eine ausführlichere Beschreibung der Ähnlichkeitsmaße findet sich öffentlich zugänglich bei PirnayDummer (2011).

\section{Artemis: Auf dem Weg zur großen Wissenslandkarte}

Artemis ist eine Weiterentwicklung von T-MITOCAR. Hierbei können aus multiplen Textquellen, z.B. aus dem Textkorpus einer Wissensdomäne, automatisch integrierte Wissenslandkartengeneriert werden. Für die Domänenmodellierung kommen verschiedene Modellierungsvarianten in Frage. Bei größeren Textbeständen kommt die „Continent by Cluster"-Methode zum Einsatz. Hierbei wird zunächst das Assoziationsnetz für jeden Einzeltext modelliert. Im Anschluss vergleicht Artemis diese Modelle hinsichtlich ihrer strukturellen und semantischen Ähnlichkeit miteinander und identifiziert in Abhängigkeit ihrer Ähnlichkeiten automatisch semantisch passende Cluster, die eine inhaltliche Zuordnung der Texte und ihrer Assoziationsmodelle $\mathrm{zu}$ verschiedenen Inhaltsbereichen einer

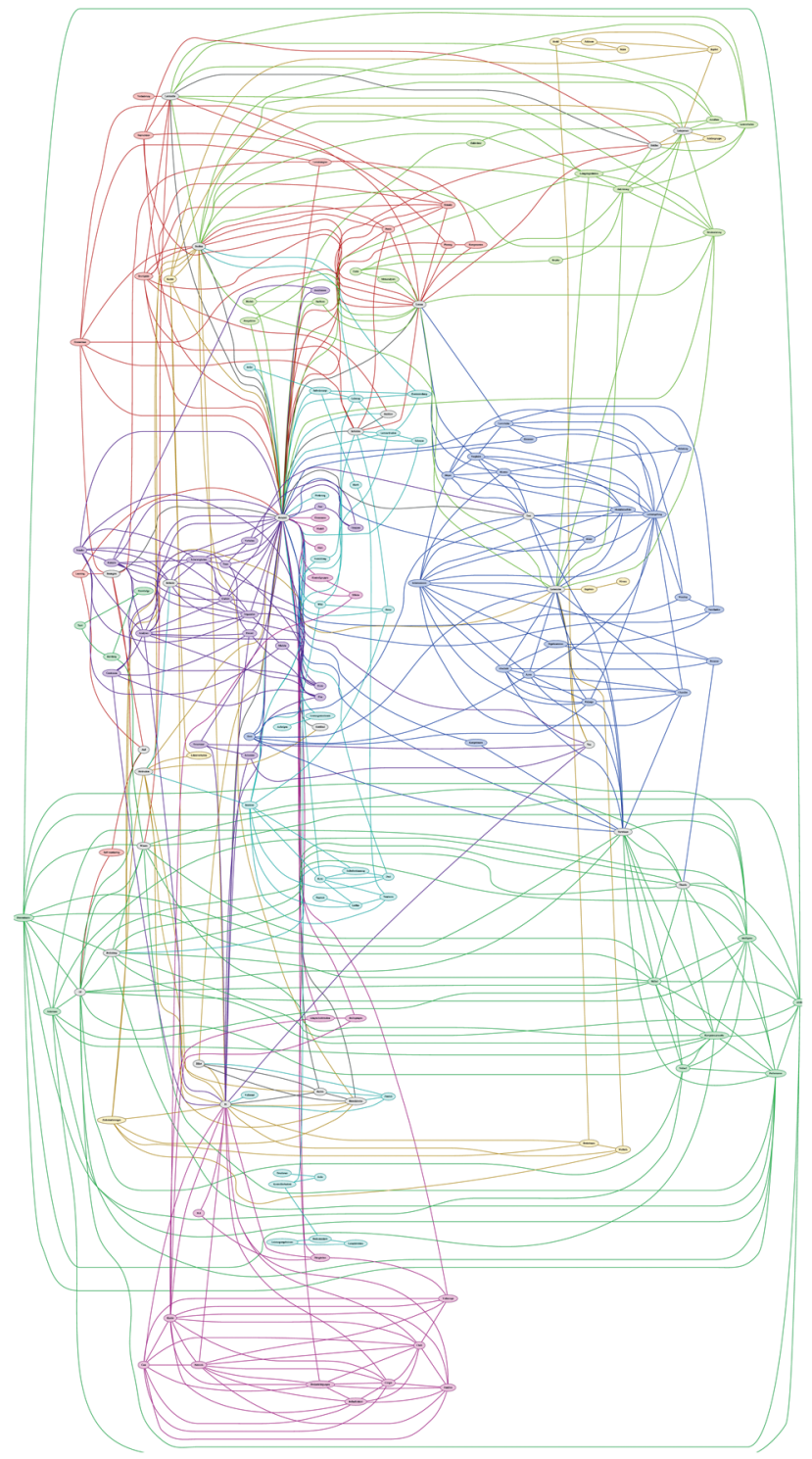

Abbildung 2: Beispiel für eine Artemis Wissenslandkarte mit acht verschiedenfarbigen Wissenskontinenten.

Domäne erlauben. Entsprechend der identifizierten Cluster werden die Einzelmodelle zu einer Wissenslandkarte integriert und aggregiert. Allein die automatische semantische Zuweisung von Texten zu Inhaltsbereichen ohne Schlagwortregister oder andere menschliche Zuordnung stellt eine potente Anwendungsklasse dar. Die automatische Zuordnung erfolgt fast fehlerlos (Lachner \& PirnayDummer, 2010).

Die entstehende Wissenslandkarte einer Domäne zeigt unter anderem, wie die gesamten analysierten Textquellen miteinander in Beziehung stehen. Dies geschieht innerhalb des ausgewählten Textkorpus über alle Buchgrenzen, Fachbereiche und Disziplinen hinweg. Besteht beispielsweise eine starke inhaltliche Assoziation zwi- 


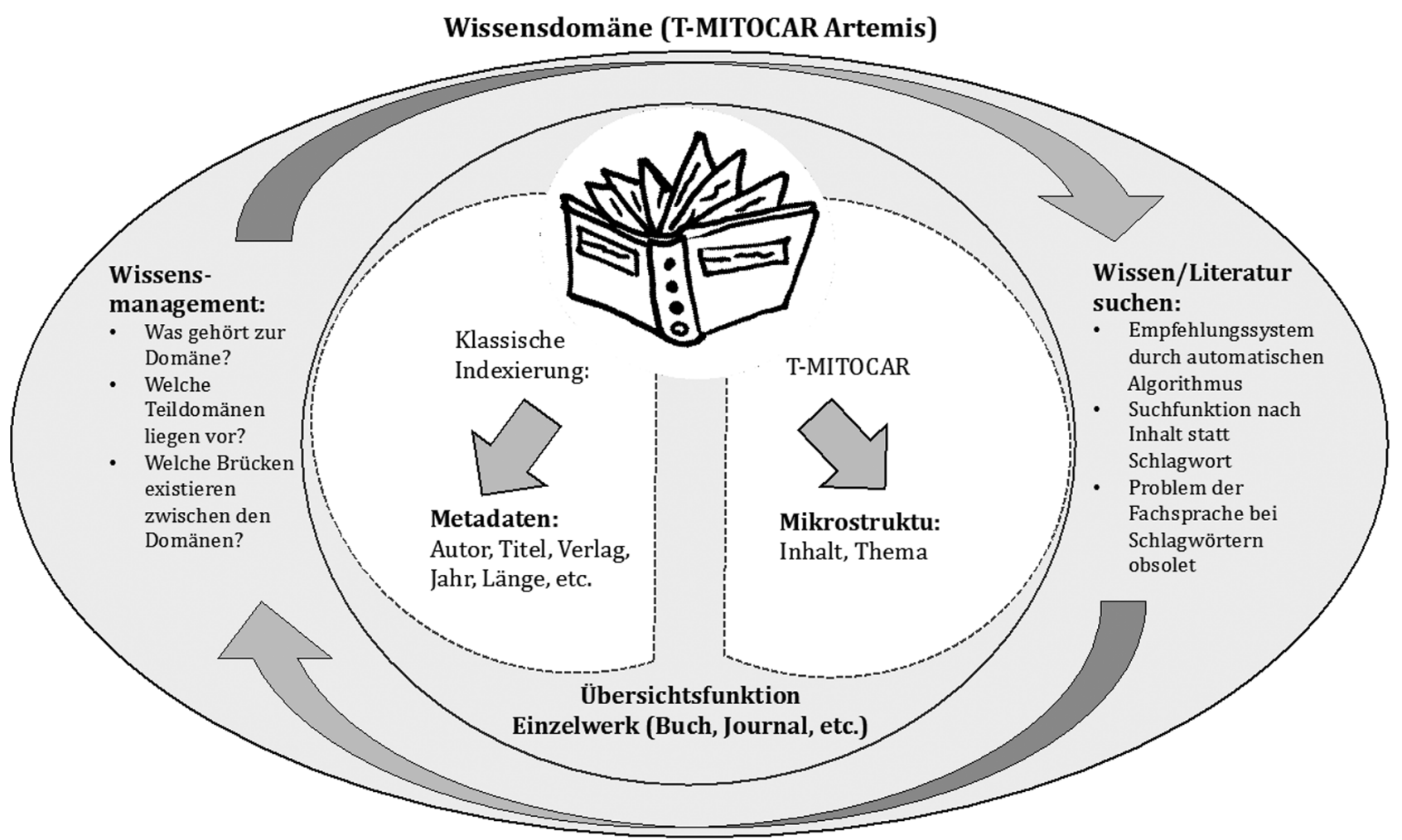

Abbildung 3: Anwendungsgebiete der Domänenmodellierung.

schen Texten verschiedener Fachbereiche, so sind diese in der Domäne auch nah beieinander verortet und befinden sich im selben Cluster. Auf diese Weise können vollständig integrierende Wissenslandkarten erstellt werden, die durch ihre schiere Größe zwar kaum als Ganzes lesbar sind, es jedoch erlauben, gezielt innerhalb der Domäne zu navigieren (Pirnay-Dummer, 2014). Die resultierende Wissenskarte kann innerhalb von Stunden aktualisiert werden. Abbildung 2 zeigt ein Beispiel für eine Wissenslandkarte mit mehreren farbigen Clustern.

\section{Anwendungsgebiete}

Als Anwendungsgebiete sind alle attraktiv, in welchen die den einzelnen Text übergreifenden Strukturen dabei helfen, nah an der psychologischen (d.h. menschlichen) Wissensabbildung Rückmeldungen für Menschen zu erzeugen. Die Technologie ist ursprünglich zur Messung und Rückmeldung von Wissen in individuellen Lernverläufen entstanden. Die Methoden erlauben ebenfalls alle Anwendung, bei denen individuell erstellte Schriften, etwa Hausund Seminararbeiten oder Essays von Studierenden, in ihrer zeitlichen Veränderung für Lehrende und Lernende abgebildet und in den Vergleich zur vorhandenen Domäne gesetzt werden. Dieser Vergleich erlaubt z. B. eine Litera- turempfehlung auf der Grundlage von bereits geschriebenen Texten - und das auf tiefer verarbeiteter Grundlage anstatt auf reiner Begriffs- und Worthäufigkeit.

Angenommen eine Institution, z.B. ein FDI, möchte seine Domäne modellieren, so erfolgt dies in mehreren Schritten. Die Einzeltexte werden zuerst mittels T-MITOCAR analysiert, anschließend wird mit T-MITOCAR Artemis ein integriertes Domänenmodell berechnet, in dem der Inhalt aller Texte miteinander verbunden wird. Das entstandene Domänenmodell kann anschließend auf verschiedene Weise eingesetzt werden, um das Wissensmanagement zu unterstützen. Abbildung 3 zeigt verschiedene Einsatzmöglichkeiten.

\section{1 Übersichtsfunktion und Wissensmanagement}

Die reine Indexierung eines Bestandes erlaubt noch keinen direkten Überblick über die Inhalte. Eine Kombination aus einem Index und einem hauseigenen Domänenmodell eröffnet nun jedoch verschiedene neue Möglichkeiten, die Literatur zu verwalten und sie anzubieten. Dies erlaubt u. a. folgende Fragen zu beantworten und für Nutzerinnen und Nutzer der Dienste aufzubereiten: Welche Inhalte decken wir mit unserem Bestand ab? Wo existie- 
ren Brücken zwischen diesen verschiedenen Inhalten und verschiedenen Fachbereichen?

Für diesen Vorgang kann ein Domänenmodell über den gesamten Bestand oder über besonders interessante aus inhaltlichen Gründen zusammengefasste Felder herangezogen werden. Ab hier wird es möglich, neu hinzutretenden Text mit der hauseigenen Domäne abzugleichen und ihn in bestehende inhaltliche Cluster einzuordnen. Auf diese Weise kann der Text im Hinblick auf seine Lage in der Domäne und seine Nachbarschaft verortet werden, womit semantische Suchen zwischen Fachbereichen ohne Verschlagwortung möglich werden. Ein solches System könnte die existierende manuelle semantische Eingliederung ergänzen, insbesondere dort, wo Fachbereichsgrenzen unterschiedliche Gliederungs- oder Hierarchisierungslogiken verwenden.

Diese Form des Wissensmanagements wäre mit der vorliegenden Methode direkt nutzbar für Bibliotheken und FDI mit ihren großen, vielseitigen Beständen.

\subsection{Navigation (Wissen anbieten)}

Die Verortung von Einzeltexten im Domänenmodell ermöglicht es, Bereiche von Interesse, ähnlich wie auf einer Landkarte, algorithmisch anzusteuern. Dokumente aus Wissensbereichen, die angrenzen und die zentrale Konzepte miteinander teilen, können ebenfalls gezielt ausgegeben werden.

Die Erstellung von verschiedenen Domänenmodellen aus unterschiedlichen Textkorpora kann inhaltliche Verbindungen oder fachliche Brücken aufzeigen (z.B. zwischen verschiedenen Fachbereichen), indem Gemeinsamkeiten und Unterschiede zwischen den einzelnen Domänenmodellen identifiziert werden. Gerade für solche Texte, deren Verschlagwortung zwischen Fachgrenzen verschieden ausfällt, könnte die Technologie Suchen vereinfachen. Suchergebnisse könnten jedoch hier nicht nur aus einer Listung und Passung von Einzeltexten gespeist werden, sondern ebenfalls auf die semantischen Cluster zurückgreifen. In diesem Fall ließe sich die Suche ggf. sogar eingrenzen, wenn Schlagworte in mehreren, inhaltlich eigentlich getrennten Bereichen auftauchen.

Die automatisierte Literaturempfehlung ermöglicht es, die Frage zu beantworten, welche anderen Texte dem Referenztext inhaltlich und strukturell am ähnlichsten sind. Gerade hierin könnte ein selbst geschriebener Text von Nutzerinnen und Nutzern die Suchanfrage darstellen: Ein T-MITOCAR-Modell wird aus dem bereits verfassten Text generiert, wonach ähnliche Cluster und ähnliche Texte gefunden werden, deren Inhalt und Struktur dem individuellen Text am nächsten kommen. Dies würde Suchanfragen substantiell vereinfachen und Iterationen, Spezifikationen und Generalisierungen verringern und manch andere die Suche präzisierende Strategie verkürzen.

\subsection{Interdisziplinarität}

Da Menschen Wörter in verschiedenen Situationen nach verschiedenen Regeln anwenden, hat ein Wort je nach Kontext unterschiedliche Funktionen. Es ist für Individuen nicht möglich alle Verwendungsmöglichkeiten $\mathrm{zu}$ überblicken, geschweige denn ihre schier unerschöpfliche Kombinatorik von außerhierarchischen Zwischenbezügen zwischen einzelnen Fächern oder Fachbereichen. Diese Problematik tritt prominent im Fachsprachengebrauch zutage und ist eine Ursache für Verständigungsprobleme im Verstehensprozess bei interdisziplinärer Kommunikation (Ballod, 2001; Felder, 2006).

Um die Domänen-Suchfunktion anzuwenden, muss zunächst ein Referenztext von Interesse (z.B. ein selbst verfasster Text) ausgewählt werden. Auf Grundlage dieses Referenztexts kann die recherchierende Person Literaturempfehlungen erhalten. Das Ergebnis ist dann nicht auf Schlagwörter ihrer eigenen Disziplin reduziert, sondern bietet die gesamte Breite der Ergebnisse an, die sich in der Domäne verbirgt, sofern sie semantisch und strukturell mit dem angelegten Referenztext Ähnlichkeiten aufweist. Dies ist mittels eines Domänenmodells möglich, das verschiedene Textkorpora enthält (in diesem Beispiel mehrerer Fachbereiche), die mittels Artemis integriert wurden.

\subsection{Kooperation mit anderen Einrichtungen}

Verfügen mehrere Institutionen über ihre hauseigene Domäne, können diese Domänenmodelle miteinander geteilt werden. Dies ist möglich, wenn mehrere Institutionen über ihre eigenen Domänenmodelle verfügen. Da lediglich das Produkt der Analyse, das Modell, ausgetauscht wird und nicht die zugrunde liegende Literatur, ist das Teilen dieser Informationen möglich, ohne dabei auf Lizenzierungen Rücksicht nehmen zu müssen. Das Teilen von Domänenmodellen kann dabei helfen, lokale Spezifika und Foki zu illustrieren, Empfehlungen mit Schwerpunkten außerhalb der eigenen Institution abzugeben. oder beim Entdecken von dynamisch gewachsenen Schwerpunkten.

Das Vorgehen kann auch dabei helfen, Berührungspunkte, Brücken und thematische Doppelungen zwischen 
den verschiedenen Domänen sichtbar zu machen. Der gezielte Austausch mit anderen Institutionen kann somit unterstützt und vereinfacht werden.

\section{Zusammenfassung}

Unterschiedliche Wissenswelten verschiedener Disziplinen werden im Prozess der Wissensdomänenmodellierung zueinander in Bezug gesetzt. Oft existieren wenig curricular verankerte Anhaltspunkte dafür, an welchen Stellen Inhalte aus verschiedenen Textquellen miteinander in Beziehung stehen (Pirnay-Dummer, im Druck). Vor allem bei stark multidisziplinären Studiengängen mit zugleich hohen Studierendenzahlen, wie in vorbereitenden Studiengängen der schulischen Lehrämter, sind Anwenderinnen und Anwender oft bei der Wissensintegration zwischen den Fachbereichen auf sich allein gestellt (z. B. Fachwissenschaft, Fachdidaktik, Pädagogik, Schulpädagogik, Psychologie). Denn die thematischen Brücken von einem Thema zum nächsten sind oftmals in der schwerer zugänglichen inhaltlichen Struktur (Mikrostruktur), also in der Prosa des Fließtexts, verborgen - und sind nicht zwangsläufig in der übersichtlicheren Makrostruktur eines Textes, d.h. in den Überschriften und Verzeichnissen, zu finden (Kintsch \& van Dijk 1978).

Dadurch, dass die Technologie um T-MITOCAR auf die Mikroebene der Texte zugreift, nimmt sie eine Strukturierung vor, die nicht durch Überschriften oder Inhaltsverzeichnisse diktiert wird, sondern das im Text enthaltene Wissen direkt verortet und feststellt, welche Texte inhaltlich aneinander angrenzen und wo Gemeinsamkeiten und Unterschiede liegen.

Auf diese Weise können Wissenslandkarten erstellt werden, die für sich selbst genommen schon eine Wissensintegration darstellen. Mit diesem methodischen Vorgehen ist es möglich, die vielen, einzelnen Inhalte einer Wissensdomäne in ein sinnvolles Wissenssystem zu integrieren, sie miteinander in kontextuelle Beziehung zu setzen und u.a. automatisierte Literaturempfehlungen auszugeben.

\section{Literatur}

Bach, E. (1989). Informal Lectures on Formal Semantics. NY: State University of New York Press.

Ballod, M. (2001). Verständliche Wissenschaft. Ein informationsdidaktischer Beitrag zur Verständlichkeitsforschung. Tübingen: Gunter Narr.

Bimba A., Idris N., Al-Hunaiyyan A., Mahmud R., Abdelaziz A., Khan S., \& Chang V., (2016) Towards knowledge modeling and mani- pulation technologies. A survey. International Journal of Information Management, 36 (6), 1-30.

Gangemi, A. (2003). Some Tools and Methodologies for Domain Ontology Building. Comparative and Functional Genomics, 4, 104-110.

Gentner, D. \& Stevens, A. L. (1983). Mental models. Hillsdale, NJ: Erlbaum.

Glaser, H. (2002) Netze des Wissens. Von Dr. Faustus zum Glasperlenspieler. In K. Beyrer \& M. Andritzky (Hrsg.), Das Netz. Sinn und Sinnlichkeit vernetzter Systeme (S. 115-124). Heidelberg: Edition Braus. Wachter Verlag.

Helbig, H. (2006). Knowledge representation and the semantics of natural language. Berlin, New York: Springer.

Hitzler P., Krötzsch, M., Rudolph, S. \& Sure, Y. (2007). Semantic Web: Grundlagen. Berlin Heidelberg: Springer-Verlag.

Johnson-Laird, P. N. (1983). Mental Models. Toward a cognitive science of language, inference and language. Cambridge: Cambridge Univ. Press.

Johnson, T. E., O’Connor, D. L., Pirnay-Dummer, P., Ifenthaler, D., Spector, J. M. \& Seel, N. M. (2006). Comparative study of mental model research methods: Relationships, among, ACSMM, SMD, MITOCAR \& DEEP methodologies. San Jose, Costa Rica: Universidad de Costa Rica.

Jonassen, D. H. \& Cho, Y. H. (2008). Externalizing mental models with mindtools. In D. Ifenthaler, P. Pirnay-Dummer \& J. M. Spector (Eds.), Understanding models for learning and instruction. Essays in honor of Norbert M. Seel. (pp. 145-159). New York: Springer.

Kintsch, W. A. \& van Dijk, T. A. (1978). Toward a model of text comprehension and production. Psychological Review, 85, 363-394

Lachner, A. \& Pirnay-Dummer, P. (2010). Model Based Knowledge Mapping. A new Approach for the Automated Graphical Representation of Organizational Knowledge. In Kinshuk, D. G. Sampson \& J. M. Spector (Eds.), Learning and Instruction in the Digital Age (pp. 79-85). New York: Springer.

Lewin, K. (1922). Das Problem der Wissensmessung und das Grundgesetz der Assoziation. Teil 1. Psychologische Forschung, 1, 191-302.

Maedche, A., Pekar, V. \& Staab, S. (2002). Ontology learning part one - on discovering taxonomic relations from the web. In Proceedings of the Web Intelligence Conference (pp. 301-322). Berlin: Springer.

Maier, R. (2005). Knowledge management systems: information and communication technologies for knowledge management. Computing Reviews, 46(1), 24.

Mertins, K. \& Seidel, H (2009). Wissensmanagement im Mittelstand. Grundlagen - Lösungen - Praxisbeispiele. Berlin Heidelberg: Springer-Verlag.

Montague, R. (1974). Formal philosophy; selected papers of Richard Montague. New Haven: Yale University Press.

Orth, R., Finke, I. \& Voigt, S. (2008). Wissen greifbar machen: Den Umgang mit Wissen beschreiben und bewerten. Ergebnisse aus den, 15 ProWis-Unternehmen. https://files.vogel.de/vogelon line/vogelonline/files/1566.pdf [15.10.2019].

Pawlowsky, P. (2019). Wissensmanagement. Oldenbourg: deGruyter. Pirnay-Dummer, P. (in press). Knowledge and structure to teach. A Model-Based Computer-Linguistic Approach to Track, Visualize, Compare and Cluster Knowledge and Knowledge Integration in Pre- Service Teachers, in Lehmann, T. (Ed.), International 
Perspectives on Knowledge Integration: Theory, Research, and Good Practice in Pre-service Teacher and Higher Education, Boston, MA: Brill Sense.

Pirnay-Dummer, P. \& Kampmann, I. (2018). Krankheitstheorien und Versorgungserwartung von Patienten sichtbar machen. In E. W. B. Hess-Lüttich (Hrsg.), Rhetorik und Medizin (Band 37, S. 176-196). Berlin: deGruyter.

Pirnay-Dummer, P. \& Seel, N. M. (2018). The Sciences of Learning. In L. Lin \& J. M. Spector (Eds.), The Sciences of Learning and Instructional Design. Constructive Articulation between Communities (pp. 8-35). New York: Routledge.

Pirnay-Dummer, P. (2015b). Linguistic analysis tools. In C. A. MacArthur, S. Graham, \& J. Fitzgerald (Eds.), Handbook of Writing Research (S. 427-442). New York: Guilford Publications.

Pirnay-Dummer, P. (2015a). Knowledge Elicitation. In J. M. Spector (Ed.), The SAGE Encyclopedia of Educational Technology (pp. 438-442). Thousand Oaks, CA: SAGE.

Pirnay-Dummer, P. (2014). Gainfully guided misconception. How Automatically Generated Knowledge Maps can Help Companies Within and Across Their Projects. In D. Ifenthaler \& R. Hanewald (Eds.), Digital knowledge maps in higher education. Technology-enhanced support for teachers and learners (S. 253-274). New York: Springer.

Pirnay-Dummer, P., Ifenthaler, D. \& Seel, N. M. (2012). Designing Model-Based Learning Environments to Support Mental Models for Learning. In D. H. Jonassen \& S. M. Land (Eds.), Theoretical Foundations of Learning Environments (pp. 55-90). New York: Routledge.

Pirnay-Dummer, P. (2012). Ontology and Semantic Web. In N. M. Seel (Ed.), Encyclopedia of the Sciences of Learning (pp. 2507-2510). New York: Springer.

Pirnay-Dummer, P. (2011). Comparison Measures of T-MITOCAR, HIMATT, and AKOVIA. http://www.pirnay-dummer.de/research/ comparison_measures_2011-03-30.pdf [30.04.2011].

Pirnay-Dummer, P. (2010b). Complete Structure Comparison. In D. Ifenthaler, P. Pirnay-Dummer \& N. M. Seel (Eds.), ComputerBased Diagnostics and Systematic Analysis of Knowledge (S. 235-258). New York: Springer.

Pirnay-Dummer, P., Ifenthaler, D. \& Spector, J. M. (2010a). Highly integrated model assessment technology and tools. Educational Technology Research and Development, 58(1), 3-18.

Pirnay-Dummer, P. \& Ifenthaler, D. (2008). Foundations and methodologies of mental models research. In D. Ifenthaler, P. PirnayDummer \& J. M. Spector (Eds.), Understanding models for learning and instruction. Essays in honor of Norbert M. Seel. (pp. 1-2). New York: Springer.

Schnotz, W. (1994). Aufbau von Wissensstrukturen. Weinheim: Beltz, Psychologie-Verl.-Union.

Schnotz, W. \& Preuss, A. (1997). Task-dependent construction of mental models as a basis for conceptual change. Aufgabenabhängige Konstruktion mentaler Modelle als Grundlage konzeptueller Veränderungen. European Journal of Psychology of Education, 12(2), 185-211.

Seel, N. M. (1991). Weltwissen und mentale Modelle. Göttingen: Hogrefe.

Smith, W. G. (1894). Mediate association. Mind, 3(11), 289-304.

Strasser, A. (2010). A functional view towards mental representations. In D. Ifenthaler, P. Pirnay-Dummer \& J. M. Spector (Eds.), Computer-Based Diagnostics and Systematic Analysis of Knowledge (pp. 15-26). New York: Springer.
Tittmann, P. (2010). Graphs and Networks. In D. Ifenthaler, P. PirnayDummer \& N. M. Seel (Eds.), Computer-Based Diagnostics and Systematic Analysis of Knowledge (pp. 177-188). New York: Springer

Villa F., Athanasiadis I. N. \& Rizzoli, A. E. (2009). Modelling with knowledge: A review of emerging semantic approaches to environmental modelling. Environmental Modelling \& Software, 24(5), 577-587.

Wells, F. L. (1911). Some properties of the free association time. Psychological review, 18, 1-24.

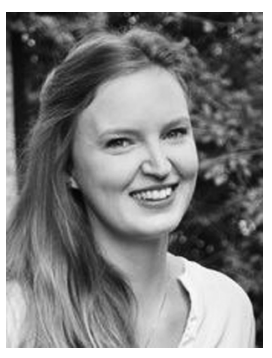

\section{Inga Kampmann, M.Sc.}

Pädagogische Psychologie

Martin-Luther-Universität Halle-Wittenberg

Franckeplatz 1, Haus 5

06110 Halle (Saale)

inga.kampmann@paedagogik.uni-halle.de

Inga Kampmann ist Psychologin (M.Sc.) und wissenschaftliche Mitarbeiterin im Arbeitsbereich Pädagogische Psychologie der MartinLuther-Universität Halle-Wittenberg. Im Rahmen ihrer Forschung in den angewandten Methoden befasste sie sich bisher mit der Erhebung und Visualisierung von Patientenvorstellungen und Versorgungserwartungen bei der Diagnose Diabetes Mellitus. Als Mitglied der interdisziplinären Projektgruppe Tech4comp zur Modellierung von akademischen Wissensdomänen (BMBF), gilt ihr besonderes Interesse der Erstellung von Entwürfen zur Rückversprachlichung von T-MITOCAR Modellvergleichsindizes und der Konzeption von maschinell synthetisierbarem Feedback für Learning Management Systeme.

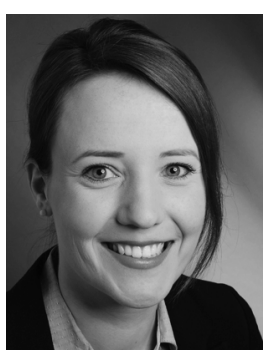

\section{Dr. phil. Inka Hähnlein}

Pädagogische Psychologie

Martin-Luther-Universität Halle-Wittenberg

Dachritzstraße 12

06108 Halle (Saale)

inka.haehnlein@paedagogik.uni-halle.de

Dr. Inka Hähnlein ist Instructional Designerin und Erziehungswissenschaftlerin. Als Post-Doc arbeitet sie seit Oktober 2018 im vom BMBF drittmittelfinanzierten tech4comp-Teilprojekt des Arbeitsbereichs Pädagogische Psychologie an der Martin-Luther-Universität Halle-Wittenberg. Hier befasst sie sich mit Wissensdiagnostik und computer-linguistischen Methoden zur Modellierung von Wissen und akademischen Wissensdomänen aus Text. Bis September 2018 forschte und lehrte sie als wissenschaftliche Mitarbeiterin und Doktorandin an der Universität Passau, wo sie in der Erziehungswissenschaft mit einer Instrumententwicklung zur Erfassung epistemologischer Überzeugungen von Lehramtsstudierenden promoviert wurde. Ihre Forschungsinteressen liegen im Bereich der empirischen Lehrund Lernforschung mit einem Schwerpunkt auf Metakognition im Lernprozess und den epistemologischen Überzeugungen von Stu- 
dierenden. Ihr besonderes Interesse gilt auch der Entwicklung von quantitativen Erhebungsinstrumenten.

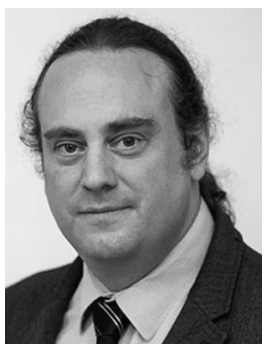

Prof. Dr. phil. habil. Pablo Pirnay-Dummer Pädagogische Psychologie

Martin-Luther-Universität Halle-Wittenberg

Franckeplatz 1, Haus 5

06099 Halle (Saale)

pablo.pirnay-dummer@paedagogik.uni-

halle.de

Prof. Dr. phil. Pablo Nicolai Pirnay-Dummer wurde zum 1. Oktober 2018 zum Professor für Pädagogische Psychologie an der Martin-Luther-Universität Halle-Wittenberg berufen. Zu seinen Spezialgebieten zählen unter anderem schriftliche und mündliche Sprachprozesse beim Lehren und Lernen. Nach dem Studium von Erziehungswissenschaft, Kognitionswissenschaft und Sprachwissenschaft des Deutschen, das er mit dem Magister Artium abschloss, wurde er Ende 2002 an der Albert-Ludwigs-Universität Freiburg promoviert. Im Oktober 2006 schloss er dort seine Habilitation mit einer Arbeit zur Sprache des Lernens ab. Anschließend nahm er Professurvertretungen an den Universitäten in Jena, Passau und Halle wahr und hatte seit Oktober 2016 die Professur für Psychologische Methodenlehre an der Medizinischen Hochschule Brandenburg Theodor Fontane (MHB) inne. Seit über zwanzig Jahren ist er außerdem Gesellschafter der in Freiburg ansässigen ParaDocks Omnimedia GbR, die Produkte und Dienstleistungen in den Bereichen Lernen und Wissen anbietet. Privat schreibt Pablo Nicolai Pirnay-Dummer Lyrik und produziert elektronische Musik. 